\title{
BASKETBALL JUMP SHOT PERFORMED BY ADULTS AND CHILDREN
}

original paper

( ) University School of Physical Education in Wrocław

DOI: https://doi.org/10.5114/hm.2018.73615

\author{
VICTOR HUGO ALVES OKAZAKI ${ }^{1}$, ANDRÉ LUIZ FÉLIX RODACKI ${ }^{2}$ \\ ${ }^{1}$ Motor Neurosciences Laboratory, Londrina State University, Londrina, Paraná, Brazil \\ ${ }^{2}$ Centre of Studies on Motor Behaviour, Federal University of Paraná, Curitiba, Paraná, Brazil
}

\section{ABSTRACT}

Purpose. The study compared the performance of basketball jump shots between children and adults.

Methods. A kinematic analysis was used to evaluate shots performance (2D; sagittal plane; $100 \mathrm{~Hz}$ ). A biomechanical model (6 points and 4 body segments) provided the shoulder, elbow, and wrist angular displacements and velocities.

Results. Children performed simultaneous movements of the shoulder-elbow-wrist joints to release the ball. Adults executed a countermovement around the elbow and wrist, but with greater constraint around the shoulder joint. This strategy may have allowed adults to achieve higher release, smaller variability, and better accuracy.

Conclusions. Differences in performance between adults and children were explained by the participants' physical characteristics and time of practice (experience).

Key words: free-throw, basketball, kinematics, motor control, biomechanics

\section{Introduction}

Shooting is one of the most important abilities in basketball [1-3], as it is the way points are obtained. Among a number of shooting techniques, jump shots provide several advantages that include accuracy, velocity, protection against opponents, and execution from several distances $[4,5]$. Therefore, regardless of the player's position [4], the jump shot has been the most efficient and commonly used shooting technique [1]. It is also considered as a complex motor skill to be learned because it depends on the performer's experience [3, 6-8] and their physical characteristics [2, 9-11].

The level of experience has been shown to affect the movement pattern of the jump shot. Novice players tend to constraint (freeze) the movement degrees of freedom as a strategy to simplify the control demands $[3,6,7]$. However, such control strategy is not efficient or skilled, possibly because it does not fully reuse elastic energy (stretch-shortening cycle) generated during the countermovement phase $[3,7]$ or the energy transferred from a proximal-to-distal sequence [12]. The energy reuse and transfer are important strategies to allow expert players to apply less strength during a shot, thereby leading to less variability and fatigue, and providing the potential for greater accuracy. Indeed, it has been proposed that mastering a sports technique involves using the elastic energy [13]. Also, experts are able to produce more consistent movements [3] and show greater stability in terms of trunk control by performing a smaller forward excursion of the centre of mass $[1,6]$. It is not known whether the variables that characterize skilled performance differ between novice and expert players in the basketball shot.

Coordinative strategies also depend on the performer's physical characteristics $[9,10,14]$. For instance, a step forward, a pronounced displacement of the centre of mass towards the basket, a great plantar flexion in the preparation phase, a large shoulder angular amplitude, a great angle, and a small ball projection of

Correspondence address: Victor Hugo Alves Okazaki, Universidade Estadual de Londrina, Departamento de Educação Física, Campus Universitário - Rodovia Celso Garcia Cid Km 380, Caixa Postal: 6001 - CEP: 86051-990, Londrina, Paraná, Brazil, e-mail: vhaokazaki@gmail.com

Received: November 18, 2016

Acepted for publication: October 26, 2017

Citation: Okazaki VHA, Rodacki ALF. Basketball jump shot performed by adults and children, Hum Mov. 2018;19(1):71-79; doi: https://doi.org/10.5114/hm.2018.73615. 
release are strategies observed in women, who are typically characterized by a smaller capacity to generate force than men [9]. Nunome et al. [14] analysed wheelchair basketball players and verified that force deficits around the wrist flexor muscles resulted in a smaller angular velocity, which was accompanied by a greater shoulder angular displacement (flexion and horizontal abduction) close to the instant of the ball release. These strategies allowed an optimization of the action of shoulder and elbow joints in an attempt to compensate the reduction of strength around the wrist flexion muscles and the absence of impulse that is generally provided by the lower limbs [14]. Other studies proved that shorter players or players with reduced capacity to jump might present a small height of ball release $[6,15]$, resulting in a steeper angle and greater velocity of the ball release $[1,10,16,17]$.

Children players typically reflect differences between these two aspects, as they are shorter and less strong, and have a lower experience level than their older counterparts. Previous studies [7] have shown that children perform shots with a larger elbow extension range and greater shoulder velocity than expert adult players. It was observed that children were unable to synchronize peak velocity and ball release. On the other hand, adults positioned the ball closer to the body and performed a countermovement around elbow and wrist joints during the shot. Differences in the movement pattern indicate the existence of two distinct strategies. The children in this study attempted to maximize the impulse applied to the ball up to release, while adults who may have more than adequate strength could use an accuracy oriented technique. Nevertheless, release variables (i.e., ball height, angle, and velocity) were not assessed. These parameters are relevant as they are the main shot determinants $[5,17]$ and may reveal differences in the performance between children and adults. Thus, the understanding of such differences may help athletes and coaches to better address the aspects that must be emphasized in training sessions leading to improved performance. However, to date, there have been no systematic efforts for the comparison of the jump shot performed by adults and children. Therefore, the present study aimed to compare the performance of basketball jump shots between children and adults. It was hypothesized that kinematic differences in the movement pattern exist when children and adults are compared.

\section{Material and methods}

\section{Participants}

The total of 30 right-handed male basketball players volunteered to participate in the study and signed (their parents in the case of children) the written informed consent form, which was approved by the local university Ethics Committee. The participants were injuryfree and did not report any inability that could interfere with the shot performance. They were assigned in one of the two following groups: adults ( $n=15$; age: $M=24.5$ years, $S D=5.4$ years; weight: $M=89.3 \mathrm{~kg}$, $S D=18.4 \mathrm{~kg}$; height: $M=1.86 \mathrm{~m}, S D=0.10 \mathrm{~m}$; basket ball experience: $M=11.5$ years of practice, $S D=6.1$ years) and children $(n=15$; age: $M=12.1$ years, $S D=$ 1.4 years; weight: $M=53.3 \mathrm{~kg}, S D=14.4 \mathrm{~kg}$; height: $M=1.62 \mathrm{~m}, S D=0.16 \mathrm{~m}$; basketball experience: $M=$ 1.3 years of practice, $S D=1.2$ years). In addition, all participants were attending regular training sessions ( 3 and 2 sessions per week, respectively for adults and children) and competitions (adults: national and state university competitions; children: regional competition level) during the period of the study.

\section{Experimental procedures}

Before the jump shot assessment, the participants were allowed to warm up for 10-20 minutes. The warmup included generalized and specific exercises in which the participants practised jump shots with minimal influence of fatigue. After the warm-up period, 9 landmarks (diameter of $1.5 \mathrm{~cm}$ ) were placed over the skin and clothes of the right body side (all players declared to be right-handed), forming a biomechanical model. Retro-reflective markers were attached at the following anatomical landmarks (Figure 1): (a) basis of the $5^{\text {th }}$ metatarsal phalange (attached on the tennis), (b) fibula lateral malleolus, (c) femur lateral epicondyle, (d) femur great trochanter, (e) iliac crest, (f) humerus great trochanter (3-5 cm bellow the acromion), (g) humerus lateral epicondyle, (h) ulna styloid process, and (i) basis of the $5^{\text {th }}$ metacarpal phalange. The link between these markers was used to form the segments of the foot (a-b), shank (b-c), leg (c-d), hip (d-e), trunk (e-f), arm (f-g), forearm (g-h), and hand (h-i). The link between the adjacent segments provided the relative angles of the following joints: ankle (foot-shank), knee (shank-leg), hip (leg-hip), shoulder (trunk-arm), elbow (arm-overarm), and wrist (overarm-hand). The analysis of the trunk segment inclination (absolute angle) was performed in function of a projected hori- 


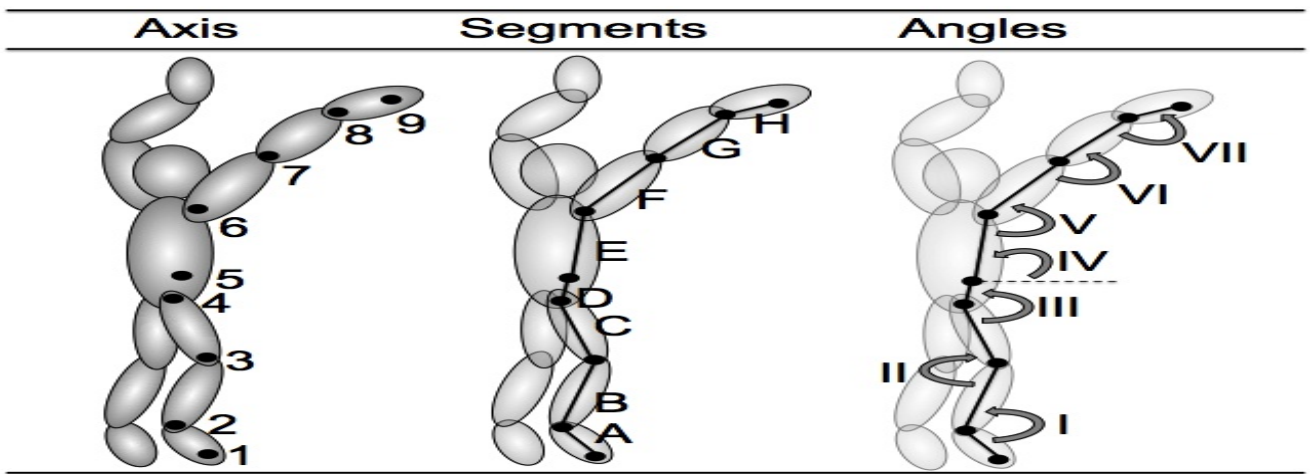

Positions of the markers: (1) basis of the $5^{\text {th }}$ metatarsal phalange, (2) fibula lateral malleolus, (3) femur lateral epicondyle, (4) femur great trochanter, (5) iliac crest, (6) humerus great trochanter, (7) humerus lateral epicondyle, (8) ulna styloid process, (9) basis of the $5^{\text {th }}$ metacarpal phalange. Segments: (A) foot, (B) leg, (C) thigh, (D) hip, (E) trunk, (F) arm, (G) forearm, (H) hand. Angles: (I) ankle, (II) knee, (III) hip, (IV) trunk, (V) shoulder, (VI) elbow, (VII) wrist.

Figure 1. Schematic representation of the biomechanical model and joint convention

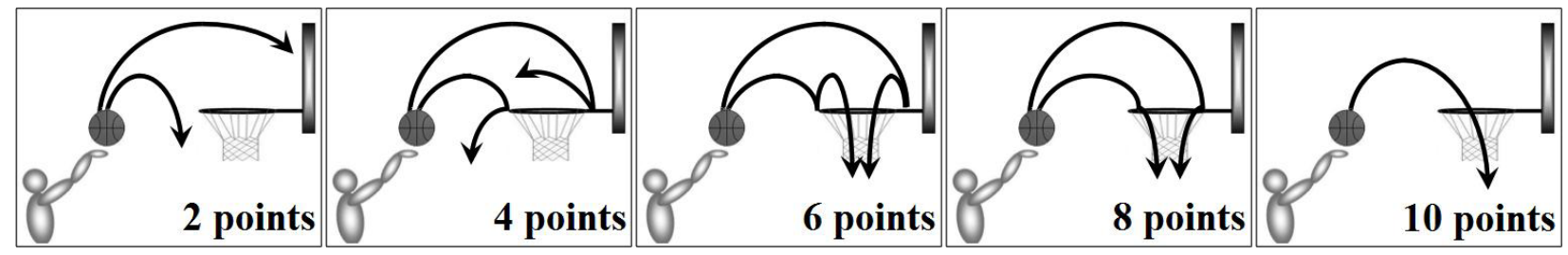

Figure 2. Representation of the scores applied to determine shot accuracy (adapted from [18])

zontal line (values above $90^{\circ}$ indicate posterior inclination).

The participants were allowed to perform 10 shots for data collection. They were requested to perform the shots right through the basket ring without using the table/backboard. During these trials, shots were video recorded and their accuracy was coded according to the schematic representation bellow (Figure 2). Adults and children performed the shots with the same ball (Penalty; model 6.4; 72-74 cm; 510-565 g) and from the free-throw line $(4.6 \mathrm{~m}$ away from the basketball ring). The kinematic characteristics of the movement were determined in a $2 \mathrm{D}$ analysis. A digital camcorder (JVC model GR-DVL 9500E, Japan) sampling at $100 \mathrm{~Hz}$ was perpendicularly positioned at approximately $8 \mathrm{~m}$ to the sagittal plane of the dominant (right) side of the participants. A rectangle of $2.5 \mathrm{~m} \times 2.0 \mathrm{~m}$ was placed on the movement plan to calibrate the kinematic data. The experimental setup is schematically represented in Figure 3. Three well-succeeded trials (score between 8 and 10) were randomly selected from the 10 performed shots for further analysis. These trials were time-normalized and merged to form an ensemble average set for each participant.

The movement start was defined as the instant in which the participant started ball elevation (from a shoulder or elbow flexion), while the movement end was specified as $0.1 \mathrm{~s}$ (10 frames) after ball release

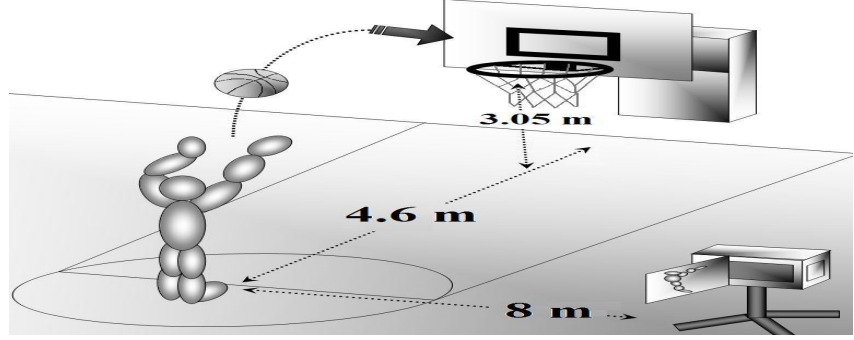

Figure 3. Schematic representation of the experimental setup

(i.e., the instant in which the ball lost hand contact). One experimenter digitized the landmarks manually using specific motion analysis software frame by frame $\left(\right.$ SIMI Motion $\left.{ }^{\circledR}\right)$. The ball centre was digitized and used to identify ball-related parameters [2].

The ball release angle, height (vertical linear displacement at release instant), and velocity (vertical, horizontal, and resultant vector components) were also analysed. The release angle was obtained by determining the angle formed by the trajectory of the ball and a horizontal line projected, calculated $0.05 \mathrm{~s}$ (5 frames) after the instant of the ball release.

Jump shot performance was analysed by ankle, knee, hip, trunk, shoulder, elbow, and wrist joints behaviour. These joints behaviour allowed to determine the following dependent variables: (a) joints' temporal series of angular displacement and velocity, (b) minimum and maximum displacements, (c) range of motion (amplitude: maximum displacement minus 


\section{HUMAN MOVEMENT}

V.H.A. Okazaki, A.L.F. Rodacki, Basketball jump shot performed by adults and children

minimum displacement), (d) angle at ball release, (e) minimum and maximum velocities, (f) angular velocities at ball release, and (g) total movement time. The centre of mass displacement and velocity (horizontal and vertical) was also analysed. The displacement of the hip joint marker was assumed to be closely related to the centre of mass displacement.

\section{Data analysis}

A recursive low-pass Butterworth filter of $4^{\text {th }}$ order was applied to reduce high frequency components with a cut-off frequency of $6 \mathrm{~Hz}$, which was determined by the residual analysis method. After filtering, joint angular displacements and velocities were calculated. Data were normalized with respect to cycle time and represented as a movement percentage. Data digitizing accuracy was determined with the use of a randomly selected video that was digitized three times and variables of the elbow calculated. The oneway repeated measures ANOVA showed no intra-trial differences and absolute differences were as small as $0.41^{\circ}(0.16 \%)$ and $1.49^{\circ} \cdot \mathrm{s}^{-1}(0.18 \%)$ for elbow angular displacement and velocity, respectively. Linear displacement (elbow) data showed errors smaller than $0.5 \%$.

\section{Statistical analysis}

Initially, all data sets were analysed with descriptive statistics (mean $[M]$ and standard deviations $[S D]$ ). The comparison of kinematic variables (spatial and temporal) and shot accuracy of adults and children was performed by applying a number of t-Student tests. The effect size was accessed through Cohen's $d$ test. The critical alpha level was set at $0.05(p<0.05)$

\section{Ethical approval}

The research related to human use has been complied with all the relevant national regulations and institutional policies, has followed the tenets of the Declaration of Helsinki, and has been approved by the authors' institutional review board or an equivalent committee.

\section{Informed consent}

Informed consent has been obtained from all individuals included in this study.

\section{Results}

Adults performed more accurate shots $(M=6.6$ points; $S D=1.0)$ in comparison with children $(M=4.6$ points;

Table 1. Mean $(S D)$ ball-related kinematics of jump shots performed by adults and children

\begin{tabular}{lccrrc}
\cline { 2 - 5 } & \multicolumn{2}{c}{ Groups } & \multicolumn{3}{c}{ Statistics } \\
\hline & \multicolumn{1}{c}{ Adults } & \multicolumn{1}{c}{ Children } & $t$ value & $p$ value & Cohen's $d$ \\
\hline Release height $(\mathrm{m})$ & $2.43(0.16)$ & $1.80(0.23)$ & 8.74 & $<0.001$ & 3.18 \\
Release angle $\left({ }^{\circ}\right)$ & $66.60(11.70)$ & $63.29(13.70)$ & 0.71 & 0.484 & 0.26 \\
Release resultant velocity $(\mathrm{m} / \mathrm{s})$ & $5.68(0.42)$ & $6.30(0.76)$ & -2.74 & 0.010 & 1.00 \\
Release horizontal velocity $(\mathrm{m} / \mathrm{s})$ & $4.34(0.54)$ & $5.23(0.74)$ & -3.80 & $<0.001$ & 1.37 \\
Release vertical velocity $(\mathrm{m} / \mathrm{s})$ & $3.63(0.40)$ & $3.46(0.54)$ & 0.94 & 0.353 & 0.21 \\
Total time $(\mathrm{s})$ & $0.774(0.111)$ & $0.672(0.167)$ & -0.40 & 0.689 & 0.72 \\
Time until ball release $(\mathrm{s})$ & $0.677(0.113)$ & $0.580(0.165)$ & -3.27 & 0.003 & 0.69 \\
Time for ball release $(\%)$ & $87.19(2.46)$ & $85.66(3.80)$ & 1.97 & 0.059 & 0.48 \\
\hline
\end{tabular}

Table 2. Mean $(S D)$ centre of mass variables for jump shots performed by adults and children

\begin{tabular}{llcrrr}
\cline { 2 - 5 } & \multicolumn{2}{c}{ Groups } & \multicolumn{2}{c}{ Statistics } \\
\cline { 2 - 6 } & \multicolumn{1}{c}{ Adults } & Children & $t$ value & $p$ value & Cohen's $d$ \\
\hline Total vertical displacement $(\mathrm{m})$ & $0.45(0.05)$ & $0.40(0.07)$ & 1.98 & 0.058 & 0.82 \\
Total horizontal displacement (m) & $0.31(0.19)$ & $0.31(0.06)$ & 0.19 & 0.843 & 0.00 \\
Release vertical displacement (m) & $0.42(0.06)$ & $0.32(0.06)$ & 3.98 & $<0.001$ & 1.67 \\
Release horizontal displacement (m) & $0.30(0.14)$ & $0.20(0.14)$ & 1.80 & 0.083 & 0.71 \\
Maximum vertical velocity (m/s) & $2.21(0.23)$ & $2.11(0.28)$ & 1.02 & 0.313 & 0.39 \\
Maximum horizontal velocity (m/s) & $0.83(0.28)$ & $0.95(0.28)$ & -1.19 & 0.244 & 0.43 \\
Release vertical velocity (m/s) & $0.22(0.68)$ & $1.29(0.44)$ & -5.13 & $<0.001$ & 1.87 \\
Release horizontal velocity (m/s) & $0.04(0.29)$ & $0.27(0.31)$ & -2.09 & 0.045 & 0.77 \\
\hline
\end{tabular}


$S D=1.5)$ and confirmed, as expected, a better expert's performance $(p<0.05)$. The more accurate jump shots executed by the adults were accompanied by a greater release height, smaller horizontal and resultant velocities, and longer duration of the release phase as compared with children (Table 1). The children group performed jump shots with greater velocity $(p<0.05)$, which was influenced by the horizontal component and a faster release shooting phase when compared with adults $(p<0.05)$.

The movements of the adults were characterized by a smaller vertical and horizontal centre of mass velocities at the ball release instant $(p<0.05)$. No differences in total vertical displacement or horizontal release velocity were detected between adults and children $(p>0.05)$. Table 2 presents the linear variables of the centre of mass among adults and children.

The trunk segment was sustained in a more erect posture in the group of adults at the ball release instant $(p<0.05)$. Adults also showed greater shoulder flexion (maximum angle, minimum angle, and angle at release) as compared with children $(p<0.05)$. In contrast, children performed jump shots with lower elbow flexion (minimum angle and amplitude; $p<0.05$ ) but with more pronounced use of the wrist joint with greater extension (minimum angle; $p<0.05$ ) and greater

Table 3. Mean (SD) joint angular displacements of jump shots performed by adults and children

\begin{tabular}{|c|c|c|c|c|c|c|c|c|}
\hline & \multirow{2}{*}{\multicolumn{3}{|c|}{ Groups }} & \multirow{3}{*}{$\begin{array}{l}p \text { value } \\
\text { Cohen's } d\end{array}$} & \multirow{2}{*}{\multicolumn{3}{|c|}{ Groups }} & \multirow{3}{*}{$\begin{array}{c}p \text { value } \\
\text { Cohen's } d\end{array}$} \\
\hline & & & & & & & & \\
\hline & Joint & Adults & Children & & Joint & Adults & Children & \\
\hline \multirow{4}{*}{$\begin{array}{l}\text { Maximum angular } \\
\text { displacement }\left(\left(^{\circ}\right)\right.\end{array}$} & Ankle & $\begin{array}{l}151.18 \\
(5.74)\end{array}$ & $\begin{array}{c}149.47 \\
(8.75)\end{array}$ & $\begin{array}{c}0.530 \\
d=0.23\end{array}$ & Shoulder & $\begin{array}{c}130.02 \\
(5.94)\end{array}$ & $\begin{array}{l}120.48 \\
(11.12)\end{array}$ & $\begin{array}{c}0.007 \\
d=1.07\end{array}$ \\
\hline & Knee & $\begin{array}{l}171.98 \\
(3.16)\end{array}$ & $\begin{array}{c}174.01 \\
(4.61)\end{array}$ & $\begin{array}{c}0.171 \\
d=0.51\end{array}$ & Elbow & $\begin{array}{l}160.62 \\
(10.17)\end{array}$ & $\begin{array}{l}164.35 \\
(8.86)\end{array}$ & $\begin{array}{c}0.293 \\
d=0.39\end{array}$ \\
\hline & Hip & $\begin{array}{l}179.78 \\
(7.87)\end{array}$ & $\begin{array}{c}174.31 \\
(6.91)\end{array}$ & $\begin{array}{c}0.052 \\
d=0.74\end{array}$ & Wrist & $\begin{array}{l}217.06 \\
(16.14)\end{array}$ & $\begin{array}{l}226.67 \\
(15.84)\end{array}$ & $\begin{array}{c}0.111 \\
d=0.60\end{array}$ \\
\hline & Trunk & $\begin{array}{l}95.15 \\
(4.36) \\
\end{array}$ & $\begin{array}{l}88.23 \\
(5.23) \\
\end{array}$ & $\begin{array}{c}0.001 \\
d=1.44\end{array}$ & & & & \\
\hline \multirow{4}{*}{$\begin{array}{l}\text { Minimum angular } \\
\text { displacement }\left({ }^{\circ}\right)\end{array}$} & Ankle & $\begin{array}{l}92.79 \\
(6.75)\end{array}$ & $\begin{array}{l}88.00 \\
(7.80)\end{array}$ & $\begin{array}{c}0.082 \\
d=0.66\end{array}$ & Shoulder & $\begin{array}{c}30.07 \\
(11.74)\end{array}$ & $\begin{array}{c}18.55 \\
(10.67)\end{array}$ & $\begin{array}{c}0.009 \\
d=1.03\end{array}$ \\
\hline & Knee & $\begin{array}{l}107.87 \\
(8.86)\end{array}$ & $\begin{array}{l}104.38 \\
(8.77)\end{array}$ & $\begin{array}{c}0.288 \\
d=0.39\end{array}$ & Elbow & $\begin{array}{l}58.17 \\
(12.48)\end{array}$ & $\begin{array}{l}71.59 \\
(16.01)\end{array}$ & $\begin{array}{c}0.016 \\
d=0.93\end{array}$ \\
\hline & Hip & $\begin{array}{l}151.41 \\
(11.84)\end{array}$ & $\begin{array}{l}142.36 \\
(12.74)\end{array}$ & $\begin{array}{c}0.053 \\
d=0.74\end{array}$ & Wrist & $\begin{array}{l}143.71 \\
(16.11)\end{array}$ & $\begin{array}{l}164.27 \\
(13.61)\end{array}$ & $\begin{array}{c}0.001 \\
d=1.38\end{array}$ \\
\hline & Trunk & $\begin{array}{c}60.93 \\
(11.56)\end{array}$ & $\begin{array}{c}56.38 \\
(14.43) \\
\end{array}$ & $\begin{array}{c}0.349 \\
d=0.35\end{array}$ & & & & \\
\hline \multirow{4}{*}{ Angular amplitude $\left({ }^{\circ}\right)$} & Ankle & $\begin{array}{l}58.39 \\
(73.70)\end{array}$ & $\begin{array}{l}61.47 \\
(6.10)\end{array}$ & $\begin{array}{c}0.234 \\
d=0.06\end{array}$ & Shoulder & $\begin{array}{l}99.94 \\
(13.73)\end{array}$ & $\begin{array}{l}101.94 \\
(14.23)\end{array}$ & $\begin{array}{c}0.699 \\
d=0.14\end{array}$ \\
\hline & Knee & $\begin{array}{l}64.12 \\
(8.63)\end{array}$ & $\begin{array}{c}69.63 \\
(11.12)\end{array}$ & $\begin{array}{c}0.140 \\
d=0.55\end{array}$ & Elbow & $\begin{array}{l}102.45 \\
(12.86)\end{array}$ & $\begin{array}{l}92.76 \\
(14.54)\end{array}$ & $\begin{array}{c}0.063 \\
d=0.71\end{array}$ \\
\hline & Hip & $\begin{array}{c}28.36 \\
(10.23)\end{array}$ & $\begin{array}{l}31.94 \\
(8.18)\end{array}$ & $\begin{array}{c}0.298 \\
d=0.39\end{array}$ & Wrist & $\begin{array}{c}73.35 \\
(17.87)\end{array}$ & $\begin{array}{c}62.40 \\
(17.12)\end{array}$ & $\begin{array}{c}0.097 \\
d=0.63\end{array}$ \\
\hline & Trunk & $\begin{array}{c}34.22 \\
(11.23)\end{array}$ & $\begin{array}{c}31.85 \\
(12.31)\end{array}$ & $\begin{array}{c}0.585 \\
d=0.20\end{array}$ & & & & \\
\hline \multirow{4}{*}{ Release angle $\left({ }^{\circ}\right)$} & Ankle & $\begin{array}{c}147.37 \\
(7.09)\end{array}$ & $\begin{array}{l}142.74 \\
(12.48)\end{array}$ & $\begin{array}{c}0.221 \\
d=0.01\end{array}$ & Shoulder & $\begin{array}{l}119.06 \\
(7.20)\end{array}$ & $\begin{array}{l}102.54 \\
(14.44)\end{array}$ & $\begin{array}{c}0.001 \\
d=1.45\end{array}$ \\
\hline & Knee & $\begin{array}{l}169.28 \\
(4.19)\end{array}$ & $\begin{array}{c}169.84 \\
(7.41)\end{array}$ & $\begin{array}{c}0.800 \\
d=0.09\end{array}$ & Elbow & $\begin{array}{c}135.47 \\
(8.26)\end{array}$ & $\begin{array}{l}139.93 \\
(17.48)\end{array}$ & $\begin{array}{c}0.378 \\
d=0.33\end{array}$ \\
\hline & Hip & $\begin{array}{l}177.96 \\
(8.18)\end{array}$ & $\begin{array}{l}171.85 \\
(7.01)\end{array}$ & $\begin{array}{c}0.036 \\
d=0.80\end{array}$ & Wrist & $\begin{array}{l}188.32 \\
(10.62)\end{array}$ & $\begin{array}{c}208.07 \\
(8.58)\end{array}$ & $\begin{array}{l}<0.001 \\
d=2.05\end{array}$ \\
\hline & Trunk & $\begin{array}{l}92.67 \\
(4.00)\end{array}$ & $\begin{array}{l}85.71 \\
(5.70)\end{array}$ & $\begin{array}{l}<0.001 \\
d=1.41\end{array}$ & & & & \\
\hline
\end{tabular}




\section{HUMAN MOVEMENT}

V.H.A. Okazaki, A.L.F. Rodacki, Basketball jump shot performed by adults and children

Table 4. Mean (SD) joint angular velocities during jump shots performed by adults and children

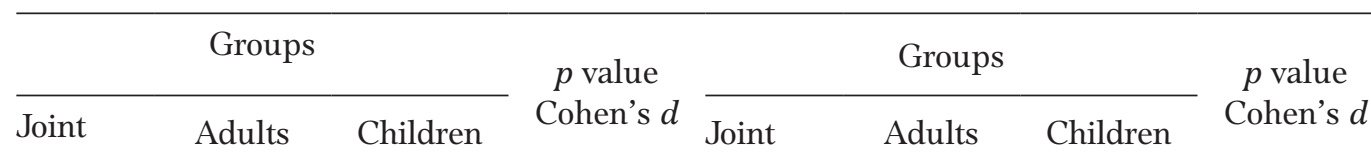

\begin{tabular}{|c|c|c|c|c|c|c|c|c|}
\hline \multirow{4}{*}{$\begin{array}{l}\text { Maximum angular } \\
\text { velocity }\left({ }^{\circ} \cdot \mathrm{s}^{-1}\right)\end{array}$} & Ankle & $\begin{array}{l}449.72 \\
(44.47)\end{array}$ & $\begin{array}{l}480.53 \\
(74.11)\end{array}$ & $\begin{array}{c}0.178 \\
d=0.50\end{array}$ & Shoulder & $\begin{array}{l}319.68 \\
(72.20)\end{array}$ & $\begin{array}{c}494.71 \\
(157.45)\end{array}$ & $\begin{array}{l}<0.001 \\
d=1.43\end{array}$ \\
\hline & Knee & $\begin{array}{l}411.86 \\
(59.03)\end{array}$ & $\begin{array}{c}512.23 \\
(112.46)\end{array}$ & $\begin{array}{c}0.005 \\
d=1.12\end{array}$ & Elbow & $\begin{array}{c}752.18 \\
(130.16)\end{array}$ & $\begin{array}{c}757.49 \\
(131.01)\end{array}$ & $\begin{array}{c}0.912 \\
d=0.04\end{array}$ \\
\hline & Hip & $\begin{array}{l}154.68 \\
(53.06)\end{array}$ & $\begin{array}{l}192.94 \\
(60.34)\end{array}$ & $\begin{array}{c}0.075 \\
d=0.67\end{array}$ & Wrist & $\begin{array}{l}203.15 \\
(82.83)\end{array}$ & $\begin{array}{c}265.41 \\
(167.62)\end{array}$ & $\begin{array}{c}0.208 \\
d=0.47\end{array}$ \\
\hline & Trunk & $\begin{array}{l}111.30 \\
(39.28)\end{array}$ & $\begin{array}{l}152.61 \\
(66.82)\end{array}$ & $\begin{array}{c}0.048 \\
d=0.75\end{array}$ & & & & \\
\hline \multirow{4}{*}{$\begin{array}{l}\text { Minimum angular } \\
\text { velocity }\left({ }^{\circ} \cdot \mathrm{s}^{-1}\right)\end{array}$} & Ankle & $\begin{array}{l}-79.53 \\
(29.45)\end{array}$ & $\begin{array}{l}-76.07 \\
(25.98)\end{array}$ & $\begin{array}{c}0.735 \\
d=0.12\end{array}$ & Shoulder & $\begin{array}{l}-63.97 \\
(52.21)\end{array}$ & $\begin{array}{c}-9.25 \\
(51.19)\end{array}$ & $\begin{array}{c}0.007 \\
d=1.05\end{array}$ \\
\hline & Knee & $\begin{array}{l}-168.37 \\
(43.04)\end{array}$ & $\begin{array}{l}-185.61 \\
(45.57)\end{array}$ & $\begin{array}{c}0.295 \\
d=0.39\end{array}$ & Elbow & $\begin{array}{l}-277.69 \\
(143.59)\end{array}$ & $\begin{array}{l}-155.23 \\
(81.17)\end{array}$ & $\begin{array}{c}0.008 \\
d=0.62\end{array}$ \\
\hline & Hip & $\begin{array}{l}-53.81 \\
(25.82)\end{array}$ & $\begin{array}{l}-76.99 \\
(40.52)\end{array}$ & $\begin{array}{c}0.072 \\
d=0.68\end{array}$ & Wrist & $\begin{array}{l}-803.49 \\
(235.59)\end{array}$ & $\begin{array}{l}-679.11 \\
(232.13)\end{array}$ & $\begin{array}{c}0.156 \\
d=0.53\end{array}$ \\
\hline & Trunk & $\begin{array}{l}-24.61 \\
(21.49)\end{array}$ & $\begin{array}{l}-47.63 \\
(38.99)\end{array}$ & $\begin{array}{c}0.055 \\
d=0.73\end{array}$ & & & & \\
\hline \multirow{4}{*}{ Release velocity $\left({ }^{0} \cdot \mathrm{s}^{-1}\right)$} & Ankle & $\begin{array}{c}42.12 \\
(118.63)\end{array}$ & $\begin{array}{c}188.84 \\
(163.59)\end{array}$ & $\begin{array}{c}0.009 \\
d=1.03\end{array}$ & Shoulder & $\begin{array}{l}267.31 \\
(89.25)\end{array}$ & $\begin{array}{c}372.05 \\
(158.41)\end{array}$ & $\begin{array}{c}0.034 \\
d=0.81\end{array}$ \\
\hline & Knee & $\begin{array}{l}17.92 \\
(93.76)\end{array}$ & $\begin{array}{c}90.26 \\
(190.54)\end{array}$ & $\begin{array}{c}0.197 \\
d=0.48\end{array}$ & Elbow & $\begin{array}{c}663.74 \\
(163.89)\end{array}$ & $\begin{array}{c}599.65 \\
(165.27)\end{array}$ & $\begin{array}{c}0.295 \\
d=0.39\end{array}$ \\
\hline & Hip & $\begin{array}{l}-21.45 \\
(47.05)\end{array}$ & $\begin{array}{c}35.02 \\
(62.41)\end{array}$ & $\begin{array}{c}0.009 \\
d=0.25\end{array}$ & Wrist & $\begin{array}{l}-709.70 \\
(221.64)\end{array}$ & $\begin{array}{l}-517.68 \\
(202.44)\end{array}$ & $\begin{array}{c}0.019 \\
d=0.90\end{array}$ \\
\hline & Trunk & $\begin{array}{l}16.40 \\
(27.95)\end{array}$ & $\begin{array}{c}35.83 \\
(57.28)\end{array}$ & $\begin{array}{c}0.247 \\
d=0.43\end{array}$ & & & & \\
\hline
\end{tabular}
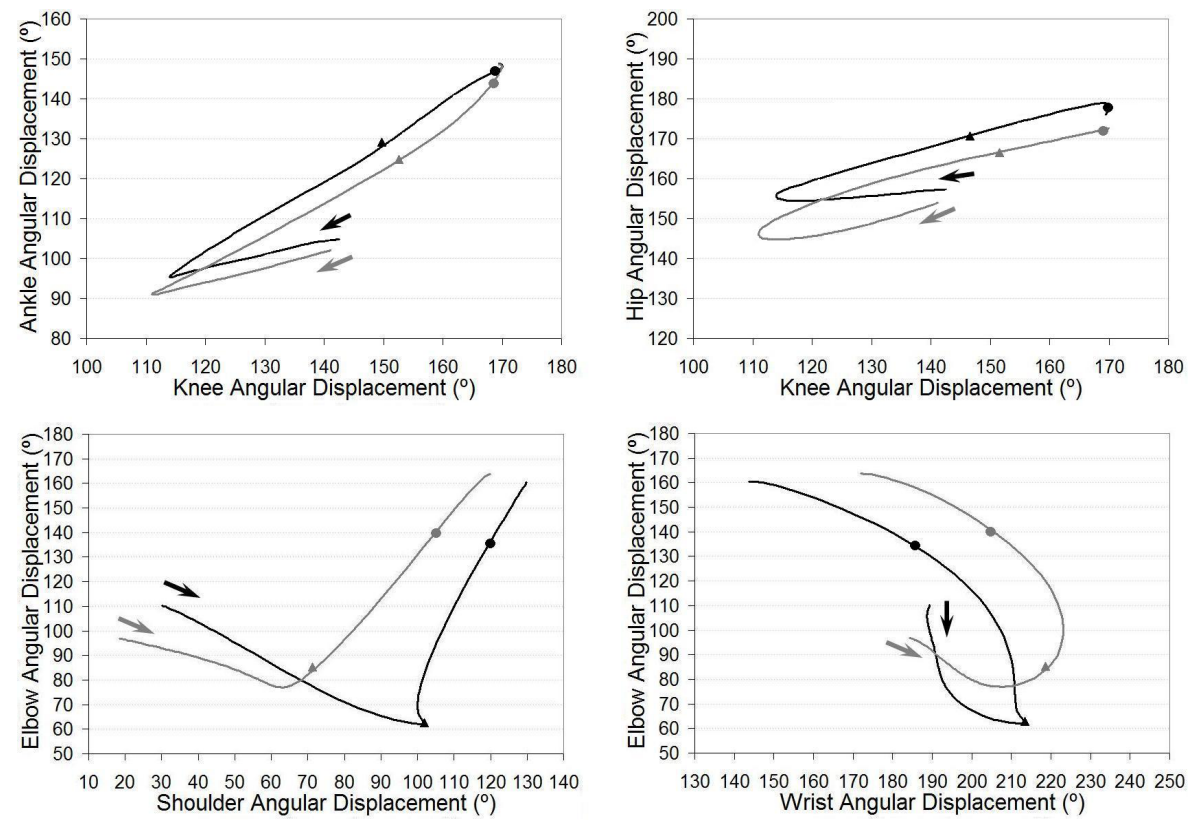

Black line - adults, gray line - children, arrows - movement direction, circles - ball release, triangles - jump at the take-off.

Figure 4. Angular displacement of adjacent joints in jump shots among adults and children (group averages) 
flexion at release $(p<0.05)$ for this joint in comparison with adults. Table 3 shows angular displacement among adults and children.

Children performed the shots with greater velocity during knee and trunk extensions and shoulder flexion when compared with adults $(p<0.05)$. At the ball release, greater velocity around ankle, hip, and shoulder joints was also observed in the group of children $(p<$ 0.05 ). Adults generated greater shoulder flexion velocity (minimum angular velocity; $p<0.05$ ) during shot preparation and release instants around the wrist when as compared with children $(p<0.05)$. Table 4 showed shots angular velocities for children and adults.

Figure 4 presents the angle-angle plots of the adjacent joints (ankle-knee, hip-knee, elbow-shoulder, elbow-wrist). It can be observed that adults applied a smaller shoulder flexion and showed a more abrupt change in the direction of the displacement pattern in the shoulder-elbow and wrist-elbow joint than children. These actions characterized a more pronounced countermovement in adults.

\section{Discussion}

Adults, as expected, showed greater accuracy in shooting when compared with children. This greater accuracy can be explained by three factors that influenced shot outcomes: (a) ball height, (b) velocity, and (c) angle at the release instant. Adults performed the jump shots with greater ball release height, where the physical characteristics of the adults (i.e., stature) obviously played a role. The greater ball release height allowed a smaller ball release velocity, as it travelled a shorter trajectory [16], which is also a way to improve accuracy [1] and has been reported to be typical of expert players [5-7]. In addition, a larger ball release angle $\left(\sim 67^{\circ}\right)$ has been considered as a relevant parameter to allow a larger incidence angle of the ball with respect to the basket, which increases the area of the basket (i.e., the virtual target $[5,11])$. The similar release angle between adults and children suggests the use of a comparable accuracy strategy. Thus, increased release height seems to be a more relevant characteristic that must be emphasized during basketball jump shot learning.

The greater height of ball release in adults can be explained by several factors, which include the greater player's stature, larger centre of mass vertical displacement, a more vertical trunk position, and a more flexed shoulder flexion. Players' stature and segment lengths are obvious influencing factors in adults, who benefit from it by the shorter distance that has to be travelled by the ball [13]. A higher elevation of the centre of mass can be achieved by greater jump heights during shooting [9]. In association, it has been suggested that synchronizing the timing of ball release with the summit of the centre of mass displacement may improve jump shot stability [12]. This strategy was identified in adults (through release vertical displacement in function of the total vertical displacement), who also released the ball closer to the highest vertical displacement as compared with children. Some authors $[8,9,11,19]$ have indicated that a ball release can occur during the ascending phase in an attempt to optimize ball propulsion, as was evidenced in children. Ball release in children not only was performed in the ascending phase but also presented greater horizontal and vertical velocities. It seems that prioritizing stability (shooting at the larger vertical displacement) is detrimental to impulse generation in novice players, while it is a strong feature among expert ones. This is in line with the current recommendation that a ball release must occur close to the highest point of the jump to ensure great release height and improve stability $[1,5]$. It has been suggested that a small backward leaning of the trunk $\left(\sim 3^{\circ}\right)$ may contribute to an increase in the ball release height [6, 19], which was confirmed as a strategy present in the expert group. The greater shoulder flexion found in adults at the ball release instant allows to increase release height $[1,9,12]$ and ball release angle $[5,20]$. These results suggest that leaning the trunk backwards and increasing shoulder flexion help to increase release height and angle, which is useful in raising shot accuracy.

Jump shots with smaller ball release height require a longer travel trajectory to the target $[2,16,17]$ and have been related with low performance shots, which are generally performed by children. This strategy includes a faster release phase that is accompanied by increased knee, trunk, and shoulder angular velocities when compared with adults. Thus, athletes with strength deficit during ball release apply a strategy that aims to increase release velocity $[8,13]$. This may explain why children emphasize lower limb joint and trunk actions in an attempt to increase ball impulse.

Children seem to adopt a pattern in which joints move in-phase (reciprocally) to generate great velocity at the ball release instant. This has been also verified in vertical jumps [21], soccer kick [22], volleyball serve [23], and basketball shots [7]. It can be interpreted as a strategy that permits to reduce information processing demand [23, 24], but has the drawback of leading to reduction of accuracy as it requires greater joint angular velocities. 
V.H.A. Okazaki, A.L.F. Rodacki, Basketball jump shot performed by adults and children

Greater variability has been reported when movement velocity is increased [25, 26], which may reduce consistency and accuracy $[1,19]$. Consequently, it has been suggested that basketball shots should be performed with reduced joint angular velocity at the ball release [5, 16, 17]. Thus, concomitant movements of shoulder-elbow-wrist during the ball release seem to increase shoulder angular velocity in children when compared with adults. This strategy prioritizes the impulse increase at the ball release and may explain the smaller accuracy in children than that observed in adults. Increased ball velocity has been identified as a characteristic of players with low capacity of strength to release the ball $[6,7,15]$. These results suggest that emphasis on shot performance must be given to strategies that minimize the velocity generation for ball propulsion.

Adults used a strategy that included a countermovement around the elbow and wrist, but with greater constraint around the shoulder joint. Probably, the countermovement around these joints allowed a greater impulse from the eccentric-concentric cycle (stretchshortening cycle) and also helped to optimize elbow propulsion action [27, 28], which has been suggested to be a common strategy in skilled performers $[3,15]$. The abrupt transition to the extension movement corroborates with the countermovement arguments proposed as a useful strategy used by adults and expert performers. Applying this strategy facilitated adults to constraint/freeze shoulder actions onto a position of great flexion to obtain greater release height.

Wrist joint has been suggested as the key aspect for movement control and accuracy in shooting $[1,10,17]$, especially because the hand is the last segment in touch with the ball before release [5]. Thus, it has been proposed that other joints are responsible for impulse generation, whereas the wrist has been pointed as more related to accuracy maintenance. The results of the present study imply that the wrist joint has a large impulse generation influence in adults and children as large angular velocities were observed on instants close to release instants. Furthermore, children used a more pronounced wrist strategy (i.e., countermovement action) than adults in an attempt to compensate for the reduced ability to generate force and power as compared with adults.

The present study verified differences between the performance of the basketball jump in adults and children. The hypothesis of the study was accepted. Children used a movement pattern that included shoulder-elbow-wrist actions more concomitantly at the ball release instant. Adults performed jump shots with a marked countermovement around elbow and wrist joints, but with a larger constraint around the shoulder, which was interpreted as a strategy to provide higher ball release height, smaller variability and, thus, greater accuracy. These performance differences can explain the better accuracy of adults in basketball jump shots when compared with children. In addition, the players' physical characteristics played a role.

The present study was limited to a $2 \mathrm{D}$ kinematic analysis. Most adult participants were not professional basketball players, and the analysis did not consider other variables that could affect the performance (shooting against an opponent, different distances from the basket, etc.). It is suggested that future studies might involve the analysis of fatigue, top level players, previous movements, release height, release velocity, and release angle.

\section{Acknowledgments}

The authors thank CAPES for the scholarship granted for the first author during the period of this research, MEC/Sesu (PET-EF) for the present granted scholarship, and CNPq for the scholarship in research productivity granted for the second author.

\section{Disclosure statement}

No author has any financial interest or received any financial benefit from this research.

\section{Conflict of interest}

The authors state no conflict of interest.

\section{References}

1. Knudson D. Biomechanics of the basketball jump shot six key teaching points. J Phys Educ Recr Dance. 1993; 64(2):67-73; doi: 10.1080/07303084.1993.10606710.

2. Malone LA, Gervais PL, Steadward RD. Shooting mechanics related to player classification on free throw success in wheelchair basketball. J Rehabil Res Dev. 2002;39(6):701-709.

3. Button C, MacLeod M, Sanders R, Coleman S. Examining movement variability in the basketball free-throw action at different skill levels. Res Q Exerc Sport. 2003; 74(3):257-269; doi: 10.1080/02701367.2003.10609090.

4. Okazaki VHA, Rodacki ALF, Sarraf TA, Dezan VH, Okazaki FHA. Diagnosis of the technical specificity of the basketball players [in Portuguese]. R Bras Cie Mov. 2004;12(4):17-24.

5. Okazaki VHA, Rodacki ALF, Satern MN. A review on the basketballjump shot. Sports Biomech. 2015;14(2):190205; doi: 10.1080/14763141.2015.1052541.

6. Hudson JL. Prediction of basketball skill using biomechanical variables. Res QExerc Sport. 1985;56(2):115121; doi: 10.1080/02701367.1985.10608445. 
7. Okazaki VHA, Rodacki ALF, Dezan VH, Sarraf TA. Coordination of the basketball jump shot performed by adults and children [in Portuguese]. Rev Bras Biomec. 2006;7(12):15-22.

8. Okazaki VHA, Lamas L, Okazaki FHA, Rodacki ALF. The effect of distance increase on the basketball shot performed by children [in Portuguese]. Motricidade. 2013;9(2):61-72; doi: 10.6063/motricidade.9(2).2668.

9. Elliott BC. A kinematic comparison of the male and female two-point and three-point jump shots in basketball. Aust J Sci Med Sport. 1992;24(4):111-118.

10. Miller S, Bartlett R. The relationship between basketball shooting kinematics, distance and playing position. J Sports Sci. 1996;14(3):243-253; doi: 10.1080/02640 419608727708.

11. Okazaki VHA, Rodacki ALF. Increased distance of shooting on basketball jump shot. J Sports Sci Med. 2012;11(2):231-237.

12. Elliott BC, White E. A kinematic and kinetic analysis of the female two point and three point jump shots in basketball. Aust J Sci Med Sport. 1989;21(2):7-11.

13. Hudson JL. Co-ordination of segments in the vertical jump. Med Sci Sports Exerc. 1986;18(2):242-251; doi: 10.1249/00005768-198604000-00015.

14. Nunome H, Doyo W, Sakurai S, Ikegmai Y, Yabe K. A kinematic study of the upper-limb motion of wheelchair basketball shooting in tetraplegic adults. J Rehabil Res Dev. 2002;39(1):63-71.

15. Hudson JL. Shooting techniques for small players. Athletic J. 1985;11:22-24.

16. Brancazio PJ. Physics of basketball. Am J Phys. 1981; 49(4):356-365; doi: 10.1119/1.12511.

17. Miller SA, Bartlett RM. The effects of increased shooting distance in the basketball jump shot. J Sports Sci. 1993;11(4):285-293; doi: 10.1080/02640419308729998.

18. Landin DK, Herbert EP, Fairweather M. The effects of variable practice on the performance of a basketball skill. Res Q Exerc Sport. 1993;64(2):263-237; doi: 10.1080/02701367.1993.10608803.

19. Satern MN. Basketball: shooting the jump shot. Strategies. 1988;1(4):9-11; doi: 10.1080/08924562.1988.10591612.

20. Okazaki VHA, Okazaki FHA, Rodacki ALF, Lima AC. Inter-individual variability on the temporal structure of the basketball shot [in Portuguese]. Motriz Rev Educ Fis. 2009;15(4):831-841.

21. Jöris HJJ, van Muyen AJE, van Ingen Schenau GJ, Kemper HCG. Force, velocity and energy flow during the overarm throw in female handball players. J Biomech. 1985;18(6):409-414; doi: 10.1016/0021-9290(85)90275-1.

22. Anderson DI, Sidaway B. Coordination changes associated with practice of a soccer kick. Res Q Exerc Sport. 1994;65(2):93-99; doi:10.1080/02701367.1994.10607603.

23. Temprado J, Della-Grasta M, Farrell M, Laurent M. A novice-expert comparison of (intra-limb) coordination subserving the volleyball serve. Hum Mov Sci. 1997; 16(5):653-676; doi: 10.1016/S0167-9457(97)00014-6.
24. Vereijken B, van Emmerik REA, Whiting HTA, Newell KM. Free(z)ing degrees of freedom in skill acquisition. J Mot Behav. 1992;24(1):133-142; doi: 10.1080/ 00222895.1992.9941608.

25. Schmidt RA, Zelaznik H, Hawkins B, Frank JS, Quinn JT Jr. Motor-output variability: a theory for the accuracy of rapid motor acts. Psychol Rev. 1979;86(5): 415-451; doi: 10.1037//0033-295X.86.5.415.

26. Meyer DE, Abrams RA, Kornblum S, Wright CE, Smith JEK. Optimality in human motor performance: ideal control of rapid aimed movements. Psychol Rev. 1988;95(3):340-370; doi:10.1037/0033-295X.95.3.340.

27. Kyröläinen H, Komi PV. The function of neuromuscular system in maximal stretch-shortening cycle exercises: comparison between power- and endurance-trained athletes. J Electromyogr Kinesiol. 1995;5(1):15-25; doi: 10.1016/S1050-6411(99)80002-9.

28. Trimble MH, Kukulka CG, Thomas RS. Reflex facilitation during the stretch-shortening cycle. J Electromyogr Kinesiol. 2000;10(3):179-187; doi: 10.1016/S10506411(00)00007-9. 